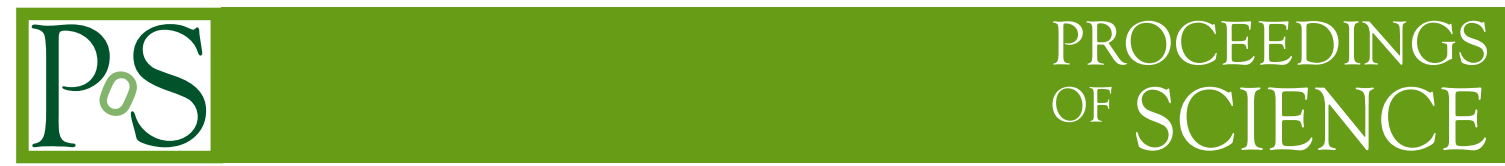

\title{
Hyperon-nucleon Interaction in Chiral EFT
}

\author{
J. Haidenbauer* \\ Institute for Advanced Simulation and Jülich Center for Hadron Physics, Forschungszentrum \\ Jülich GmbH, D-52425 Jülich, Germany \\ E-mail: j.haidenbauer@fz-juelich.de
}

\begin{abstract}
I report on recent progress in the description of the hyperon-nucleon interaction within chiral effective field theory. In particular, first results for the hyperon-nucleon interaction to next-toleading order are presented.
\end{abstract}

The 7th International Workshop on Chiral Dynamics, August 6 -10, 2012

Jefferson Lab, Newport News, Virginia, USA

* Speaker. 


\section{Introduction}

Chiral effective field theory (EFT) as proposed by Weinberg [1,2] more than two decades ago turned out to be a rather powerful tool for the derivation of nuclear forces. In this scheme there is an underlying power counting which allows to improve calculations systematically by going to higher orders in a perturbative expansion. In addition, it is possible to derive two- and corresponding three-nucleon forces as well as external current operators in a consistent way. Over the last decade or so it has been demonstrated that the nucleon-nucleon $(N N)$ interaction can be described to a high precision within the chiral EFT approach $[3,4]$. Following the original suggestion of Weinberg, in these works the power counting is applied to the $N N$ potential rather than to the reaction amplitude. The latter is then obtained from solving a regularized Lippmann-Schwinger equation for the derived interaction potential. The $N N$ potential contains pion-exchanges and a series of contact interactions with an increasing number of derivatives to parameterize the shorter ranged part of the $N N$ force. For reviews we refer the reader to Refs. [5, 6, 7].

In this contribution I report results of ongoing investigations by the groups in Bonn-Jülich and Munich on the baryon-baryon interaction involving strange baryons, performed within chiral EFT $[8,9,10,11,12,13]$. In those studies the same scheme that has been applied in Ref. [4] to the $N N$ interaction is utilized for the strangeness $S=-1$ to -4 sectors. Here I consider specifically baryon-baryon systems with $S=-1$, namely $\Lambda N$ and $\Sigma N$. In this case the extension of our leadingorder (LO) study [8] to next-to-leading order (NLO) is in progress [12] and a first glimpse on the (still preliminary) achieved results for the $\Lambda N$ and $\Sigma N$ interactions will be given.

At LO in the power counting, as considered in the aforementioned investigations $[8,9,10]$, the baryon-baryon potentials involving strange baryons consist of four-baryon contact terms without derivatives and of one-pseudoscalar-meson exchanges, analogous to the $N N$ potential of [4]. The potentials are derived using constraints from SU(3) flavor symmetry. At NLO one gets contributions from two-pseudoscalar-meson exchange diagrams and from four-baryon contact terms with two derivatives [4].

The paper is structured as follows: In Sect. 2 a short overview of the chiral EFT approach is provided. In Sect. 3 results for the $\Lambda N$ - and $\Sigma N$ interactions obtained to NLO are presented. The paper ends with a brief Summary.

\section{Formalism}

The derivation of the chiral baryon-baryon potentials for the strangeness sector at $\mathrm{LO}$ using the Weinberg power counting is outlined in Refs. [8, 10, 14]. Details for the NLO case will be presented in a forthcoming paper [13], see also [11]. The LO potential consists of four-baryon contact terms without derivatives and of one-pseudoscalar-meson exchanges while at NLO contact terms with two derivatives arise, together with contributions from (irreducible) two-pseudoscalarmeson exchanges.

The spin- and momentum structure of the potentials resulting from the contact terms to LO is given by

$$
V_{B B \rightarrow B B}^{(0)}=C_{S ; B B \rightarrow B B}+C_{T ; B B \rightarrow B B}\left(\sigma_{1} \cdot \sigma_{2}\right)
$$


in the notation of [4] where the $C_{i ; B B \rightarrow B B}$ 's are so-called low-energy coefficients (LECs) that need to be determined by a fit to data. Due to the imposed $\mathrm{SU}(3)_{\mathrm{f}}$ constraints there are only five independent LECs for the $N N$ and the $Y N$ sectors together, as described in Ref. [8] where also the relations between the various $C_{i ; B B \rightarrow B B}$ 's are given. A sixth LEC is, however, present in the strangeness $S=-2$ channels with isospin $I=0$.

In next-to-leading order one obtains the following spin- and momentum structure:

$$
\begin{aligned}
V_{B B \rightarrow B B}^{(2)} & =C_{1} \mathbf{q}^{2}+C_{2} \mathbf{k}^{2}+\left(C_{3} \mathbf{q}^{2}+C_{4} \mathbf{k}^{2}\right)\left(\sigma_{1} \cdot \sigma_{2}\right)+\frac{i}{2} C_{5}\left(\sigma_{1}+\sigma_{2}\right) \cdot(\mathbf{q} \times \mathbf{k}) \\
& +C_{6}\left(\mathbf{q} \cdot \sigma_{1}\right)\left(\mathbf{q} \cdot \sigma_{2}\right)+C_{7}\left(\mathbf{k} \cdot \sigma_{1}\right)\left(\mathbf{k} \cdot \sigma_{2}\right)+\frac{i}{2} C_{8}\left(\sigma_{1}-\sigma_{2}\right) \cdot(\mathbf{q} \times \mathbf{k}) .
\end{aligned}
$$

The transferred and average momentum, $\mathbf{q}$ and $\mathbf{k}$, are defined in terms of the final and initial centerof-mass (c.m.) momenta of the baryons, $\mathbf{p}^{\prime}$ and $\mathbf{p}$, as $\mathbf{q}=\mathbf{p}^{\prime}-\mathbf{p}$ and $\mathbf{k}=\left(\mathbf{p}^{\prime}+\mathbf{p}\right) / 2$. The $C_{i}{ }^{\prime} \mathrm{s}$ (actually $C_{i ; B B \rightarrow B B}$ 's) are additional LECs. Performing a partial wave projection and imposing again $\mathrm{SU}(3)_{\mathrm{f}}$ symmetry one finds that in case of the $Y N$ interaction there are eight new LECs entering the $S$-waves and $S$ - $D$ transitions, respectively, and ten coefficients in the $P$-waves. There are further (four) LECs that contribute only to the $S=-2$ baryon-baryon sector.

The spin-space part of the one-pseudoscalar-meson-exchange potential is similar to the static one-pion-exchange potential (recoil and relativistic corrections give higher order contributions) and follows from the $\mathrm{SU}(3)_{\mathrm{f}}$ invariant pseudoscalar-meson-baryon interaction Lagrangian with the appropriate symmetries as discussed in [8]:

$$
V_{B_{1} B_{2} \rightarrow B_{1}^{\prime} B_{2}^{\prime}}^{O B E}=-f_{B_{1} B_{1}^{\prime} P} f_{B_{2} B_{2}^{\prime} P} \frac{\left(\sigma_{1} \cdot \mathbf{q}\right)\left(\sigma_{2} \cdot \mathbf{q}\right)}{\mathbf{q}^{2}+M_{P}^{2}} .
$$

Here, $M_{P}$ is the mass of the exchanged pseudoscalar meson. The coupling constants $f_{B B^{\prime} P}$ at the various baryon-baryon-meson vertices are fixed by the imposed $\mathrm{SU}(3)$ constraints and tabulated, e.g., in [8]. They can be expressed in terms of $f \equiv g_{A} / 2 F_{\pi} \equiv f_{N N \pi}\left(g_{A}=1.26, F_{\pi}=92.4 \mathrm{MeV}\right)$ and $\alpha$, the so-called $F /(F+D)$-ratio, for which we adopted the $\mathrm{SU}(6)$ value $(\alpha=0.4)$. Note that we use the physical masses of the exchanged pseudoscalar mesons. Thus, the explicit SU(3) breaking reflected in the mass splitting between the pseudoscalar mesons is taken into account. The $\eta$ meson was identified with the octet $\eta\left(\eta_{8}\right)$ and its physical mass was used. The two-pseudoscalar-mesonexchange potential can be found in Refs. [11, 13].

The reaction amplitudes are obtained from the solution of a coupled-channels LippmannSchwinger (LS) equation for the interaction potentials:

$$
T_{v^{\prime \prime} v^{\prime}}^{\rho^{\prime \prime} \rho^{\prime}, J}\left(p^{\prime \prime}, p^{\prime} ; \sqrt{s}\right)=V_{v^{\prime \prime} v^{\prime}}^{\rho^{\prime \prime} \rho^{\prime}, J}\left(p^{\prime \prime}, p^{\prime}\right)+\sum_{\rho, v} \int_{0}^{\infty} \frac{d p p^{2}}{(2 \pi)^{3}} V_{v^{\prime \prime} v}^{\rho^{\prime \prime} \rho, J}\left(p^{\prime \prime}, p\right) \frac{2 \mu_{v}}{q_{v}^{2}-p^{2}+i \eta} T_{v v^{\prime}}^{\rho \rho^{\prime}, J}\left(p, p^{\prime} ; \sqrt{s}\right) \text {. }
$$

The label $v$ indicates the particle channels and the label $\rho$ the partial wave. $\mu_{v}$ is the pertinent reduced mass. The on-shell momentum in the intermediate state, $q_{v}$, is defined by $\sqrt{s}=$ $\sqrt{m_{B_{1, v}}^{2}+q_{v}^{2}}+\sqrt{m_{B_{2, v}}^{2}+q_{v}^{2}}$. Relativistic kinematics is used for relating the laboratory energy $T_{\text {lab }}$ of the hyperons to the c.m. momentum.

We solve the LS equation in the particle basis, in order to incorporate the correct physical thresholds. Depending on the total charge up to three baryon-baryon channels can couple. The 

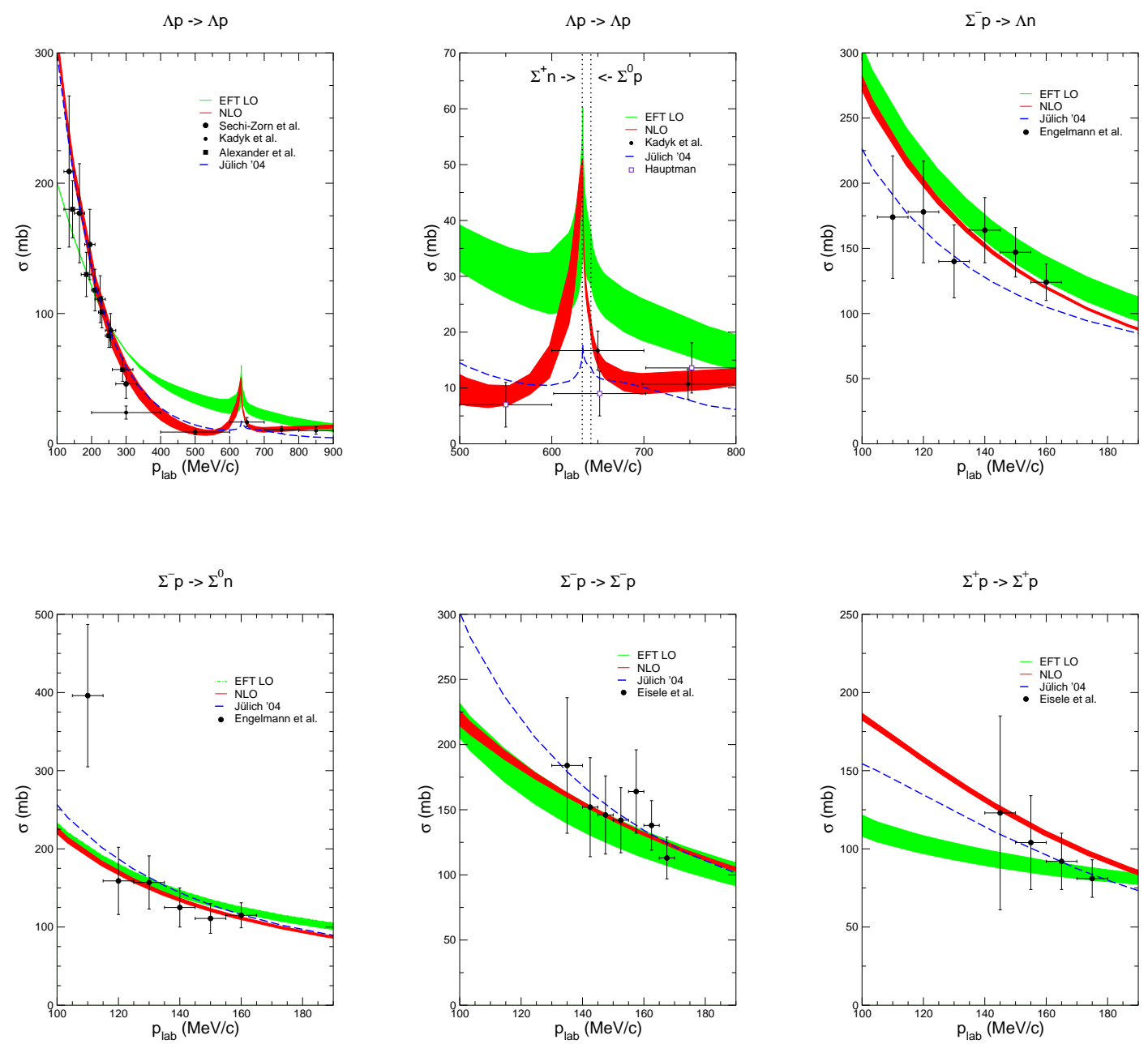

Figure 1: Total cross sections for $\Lambda p \rightarrow \Lambda p, \Sigma^{-} p \rightarrow \Lambda n, \Sigma^{-} p \rightarrow \Sigma^{0} n, \Sigma^{-} p \rightarrow \Sigma^{-} p$ and $\Sigma^{+} p \rightarrow \Sigma^{+} p$ as a function of $p_{\text {lab. }}$. The green (grey) band shows the chiral EFT results to LO for variations of the cut-off in the range $\Lambda=550 \ldots 700 \mathrm{MeV}$, while the red (black) band are results to NLO for $\Lambda=500 \ldots .700 \mathrm{MeV}$. The dashed curve is the result of the Jülich '04 [16] meson-exchange potential.

Coulomb interaction is taken into account appropriately via the Vincent-Phatak method [15]. The potentials in the LS equation are cut off with a regulator function, $\exp \left[-\left(p^{4}+p^{4}\right) / \Lambda^{4}\right]$, in order to remove high-energy components of the baryon and pseudoscalar meson fields [4]. We consider cut-off values in the range $500, \ldots, 700 \mathrm{MeV}$, similar to what was used for chiral $N N$ potentials [4].

\section{Results for the $\Lambda N$ and $\Sigma N$ systems}

The imposed SU(3) flavor symmetry implies that at LO five independent LECs contribute to the $Y N$ interaction [8]. These five contact terms were determined in [8] by a fit to the $Y N$ scattering data. Already in that scenario a fairly reasonable description of the 35 low-energy $Y N$ scattering 
data could be achieved for cutoff values $\Lambda=550, \ldots, 700 \mathrm{MeV}$ and for natural values of the LECs. At NLO there are eight new contact terms contributing to the $S$-waves and the ${ }^{3} S_{1}-{ }^{3} D_{1}$ transition, and ten in the $P$-waves. Once again the corresponding LECs were fixed by fitting to the data. The results obtained at NLO are presented in Fig. 1 (black (red) bands), together with those at LO (grey (green) bands). The bands represent the variation of the cross sections based on chiral EFT within the considered cutoff region. For comparison also results for the Jülich '04 [16] meson-exchange models are shown (dashed line).

Obviously, and as expected, the energy dependence exhibited by the data can be significantly better reproduced within our NLO calculation. This concerns in particular the $\Lambda p$ channel where now the theoretical results are well in line with the data even up to and beyond the $\Sigma N$ threshold. There is also a noticeable improvement in case of the $\Sigma^{+} p$ interaction. Furthermore, one can see that the dependence on the cutoff mass is strongly reduced in the NLO case.

Note that in case of $\mathrm{LO}$ as well as at $\mathrm{NLO}$ no SU(3) $)_{\mathrm{f}}$ constraints from the $N N$ sector were imposed in the fitting procedure. The leading order $\mathrm{SU}(3)_{\mathrm{f}}$ breaking in the one-boson exchange diagrams (coupling constants) is ignored.

Besides an excellent description of the $Y N$ data the chiral EFT interaction also yields a correctly bound hypertriton, see Table 1 . Indeed this binding energy had to be included in the fitting procedure because otherwise it would have not been possible to fix the relative strength of the (S-wave) singlet- and triplet contributions to the $\Lambda p$ interaction. Table 1 lists also results for two meson-exchange potentials, namely for the Jülich '04 model [16] and the Nijmegen NSC97f potential [17], which both reproduce the hypertriton binding energy correctly. Obviously, the scattering lengths predicted at NLO are larger than those obtained at LO and now similar to the values of the meson-exchange potentials. The $\Sigma^{+} p$ scattering length in the ${ }^{3} S_{1}$ partial wave is positive, as it was already the case for our LO potential, indicating a repulsive interaction in this channel.

\begin{tabular}{|c|c|c|cc|c|}
\hline & $\begin{array}{c}\text { EFT LO } \\
{[8]}\end{array}$ & EFT NLO & $\begin{array}{c}\text { Jülich '04 } \\
{[16]}\end{array}$ & $\begin{array}{c}\text { NSC97f } \\
{[17]}\end{array}$ & experiment \\
\hline \hline$\Lambda[\mathrm{MeV}]$ & $550 \cdots 700$ & $500 \cdots 700$ & & & \\
\hline$a_{s}^{\Lambda p}$ & $-1.90 \cdots-1.91$ & $-2.88 \cdots-2.89$ & -2.56 & -2.51 & $-1.8_{-4.2}^{+2.3}$ \\
$a_{t}^{\Lambda p}$ & $-1.22 \cdots-1.23$ & $-1.59 \cdots-1.61$ & -1.66 & -1.75 & $-1.6_{-0.8}^{+1.1}$ \\
\hline$a_{s}^{\Sigma^{+} p}$ & $-2.24 \cdots-2.36$ & $-3.90 \cdots-3.83$ & -4.71 & -4.35 & \\
$a_{t}^{\Sigma^{+} p}$ & $0.70 \cdots 0.60$ & $0.51 \cdots 0.47$ & 0.29 & -0.25 & \\
\hline \hline$\left({ }_{\Lambda}^{3} \mathrm{H}\right) E_{B}$ & $-2.34 \cdots-2.36$ & $-2.31 \cdots-2.34$ & -2.27 & -2.30 & $-2.354(50)$ \\
\hline
\end{tabular}

Table 1: The $Y N$ singlet (s) and triplet (t) scattering lengths (in fm) and the hypertriton binding energy, $E_{B}$ (in $\mathrm{MeV}$ ).

Calculations for the four-body hypernuclei ${ }_{\Lambda}^{4} \mathrm{H}$ and ${ }_{\Lambda}^{4} \mathrm{He}$ based on those interactions are reported in Ref. [18].

\section{Summary}

Chiral effective field theory, successfully applied in Refs. [3, 4] to the $N N$ interaction, also 
works well for the baryon-baryon interactions in the strangeness $S=-1(\Lambda N-\Sigma N)[8,12]$ and $S=-2(\Lambda \Lambda-\Xi N-\Sigma \Sigma)$ sectors [9]. As shown in our earlier work, already at leading order the bulk properties of the $\Lambda N$ and $\Sigma N$ systems can be reasonably well accounted for. The new results for the $Y N$ interaction presented here, obtained to next-to-leading order in the Weinberg counting, look very promising. First there is a visible improvement in the quantitative reproduction of the available data on $\Lambda N$ and $\Sigma N$ scattering and, secondly, the dependence on the regularization scheme is strongly reduced as compared to the LO result. Indeed the description of the $Y N$ system achieved at NLO is now on the same level of quality as the one by the most advanced meson-exchange $Y N$ interactions.

\section{Acknowledgements}

I would like to thank N. Kaiser, U.-G. Meißner, A. Nogga, S. Petschauer, and W. Weise for collaborating on the topic covered by my talk. Work supported in part by DFG and NSFC (CRC 110).

\section{References}

[1] S. Weinberg, Phys. Lett. B 251 (1990) 288.

[2] S. Weinberg, Nucl. Phys. B 363 (1991) 3.

[3] D. R. Entem, R. Machleidt, Phys. Rev. C 68 (2003) 041001.

[4] E. Epelbaum, W. Glöckle, U.-G. Meißner, Nucl. Phys. A 747 (2005) 362.

[5] P. F. Bedaque, U. van Kolck, Annu. Rev. Nucl. Part. Sci. 52 (2002) 339.

[6] E. Epelbaum, Prog. Part. Nucl. Phys. 57 (2006) 654.

[7] E. Epelbaum, H. -W. Hammer and U.-G. Meißner, Rev. Mod. Phys. 81 (2009) 1773.

[8] H. Polinder, J. Haidenbauer and U.-G. Meißner, Nucl. Phys. A 779 (2006) 244.

[9] H. Polinder, J. Haidenbauer and U.-G. Meißner, Phys. Lett. B 653 (2007) 29.

[10] J. Haidenbauer, U.-G. Meißner, Phys. Lett. B 684 (2010) 275.

[11] S. Petschauer, diploma thesis, TU Munich, 2011.

[12] J. Haidenbauer, arXiv:1301.1141 [nucl-th].

[13] J. Haidenbauer et al., arXiv:1304.5339 [nucl-th], Nucl. Phys. A, in press.

[14] J. Haidenbauer, U.-G. Meißner, A. Nogga and H. Polinder, Lect. Notes Phys. 724 (2007) 113.

[15] C.M. Vincent and S.C. Phatak, Phys. Rev. C 10 (1974) 391.

[16] J. Haidenbauer, U.-G. Meißner, Phys. Rev. C 72 (2005) 044005.

[17] T. A. Rijken, V. G. J. Stoks, Y. Yamamoto, Phys. Rev. C 59 (1999) 21.

[18] A. Nogga, in XI International Conference on Hypernuclear and Strange Particle Physics, Barcelona, October 1-5, 2012, Nucl. Phys. A, in press. 